\title{
Pitch Detection of Musical Sounds Noticing Minimum Output of Parallel Connected Comb Filters
}

\author{
YOSHIAKI TADOKORO, TAKEO MORITA and MICHIRU YAMAGUCHI \\ Department of Information and Computer Sciences \\ Toyohashi University of Technology \\ Hibarigaoka 1-1, Tempaku, Toyohashi, 441-8580 \\ JAPAN \\ http://www.signal.tutics.tut.ac.jp
}

\begin{abstract}
This paper proposes a simple pitch detection method using the parallel connected comb filters. We can know the pitches of a musical sound by detecting the smaller outputs in twelve comb filters connected in parallel. Each comb filter has zero points corresponding to each pitch and its harmonic frequencies, and so the outputs of the comb filters corresponding to input pitch frequencies have smaller frequency components, and show smaller outputs than other comb filters' ones. We could detect the pitches with the probability of $98.2 \%$ for double tones and $91.1 \%$ for triple tones.
\end{abstract}

Key-Words: - Transcription, Comb filter, Parallel connected comb filters, Minimum output

\section{Introduction}

Musical transcription is necessary in the musicology field and also a significant problem in machine perception [1]-[3]. The pitch detection is a most important role for transcription. A musical instrument sound is composed of a fundamental frequency (pitch frequency) corresponding to each tone and its harmonic frequencies. But, these harmonic components are changing complicatedly depending on each tone and each musical instrument. This is the reason that the pitch detection is difficult, especially in polyphonic music played by several different instruments.

Many methods that have been presented for the pitch detection are based on the extraction of the fundamental frequencies using the frequency analysis such as the fast Fourier transform (FFT) and adaptive filtering [4]-[7]. We proposed a unique method for the pitch detection that is based on the elimination of the pitch and its harmonic frequencies using a comb filter $\left(H(z)=1-z^{-N}\right)$ [8][9]. The proposed method can simply determine the pitches of the sound played by several different instruments by detecting the zero outputs of the cascaded twelve comb filters as shown in Fig.1, independently of musical instruments [9]. In Fig.1, $H_{p, q}(z)$ shows a comb filter for octave $\mathrm{p}(\# \mathrm{p})$ and q pitch name (tone : \#p, q). But this method does not work well when the played musical sounds have some frequency deviations from the nominal frequency values corresponding to the tones and some noises. Because, in such cases, signal frequency components are amplified and the outputs of comb filters do no become zero, as realizing the total magnitude characteristic of the cascaded twelve comb filters shown in Fig.2. This is not suitable for the pitch detection system using the zero output.

To overcome these problems, we proposed another

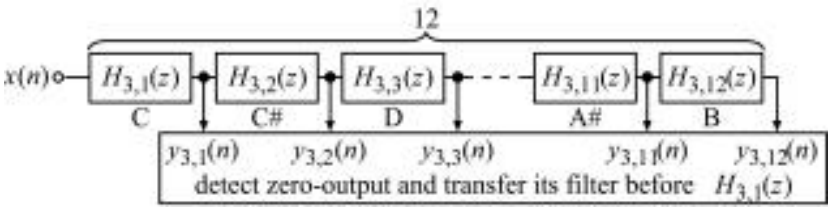

Fig.1 Pitch detection system based on cascaded twelve comb filters.

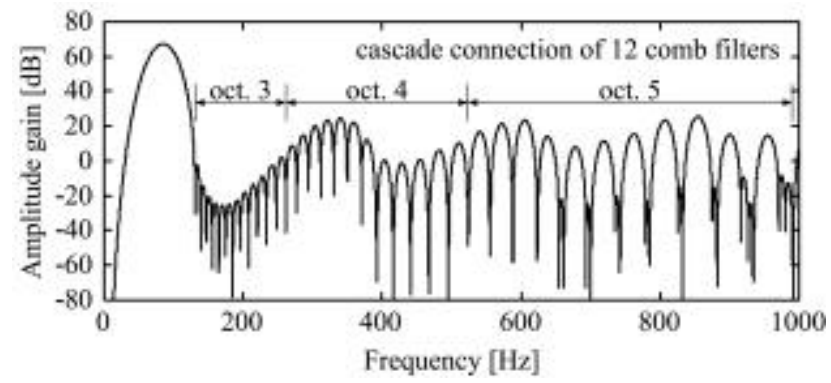

Fig.2 Total magnitude characteristic of the cascaded twelve comb filters : $\prod_{q=1}^{12} H_{3, q}(z)$. 
two method. One of them is based on the cascade connected adaptive comb filters where the necessary number of the cascaded comb filters is the minimum number that is necessary for a polyphony input, for example, three adaptive comb filters for the tones less than triple tones [10]. The other is the method that uses twelve comb filters connected in parallel and singular value decomposition (SVD) processing as shown in Fig.3 [11]. In this parallel method, the comb filter outputs corresponding to the input pitch frequencies has smaller frequency components than other comb filter outputs. We can know the smaller frequency components using the SVD processing. But this SVD processing has $N^{3}$ multiplications for $(N \times N)$ matrix data of the input musical sound.

In this paper, we propose a simple method to improve this computational complexity in the parallel comb filter method using the SVD processing. We suppose we can detect the outputs having smaller frequency components by noticing the amplitude of the comb filter outputs. That is, it is expected that the amplitude of the comb filter having the smaller frequency components is smaller than other outputs. We assume the tones from octaves 3 to 5 in this paper, i.e., 36 tones.

\section{Principle of Proposed Method}

The comb filter $H_{p, q}(z)$ for octave $\mathrm{p}$ and $\mathrm{q}$ pitch name can be written by

$$
H_{p, q}(z)=1-z^{-N_{q}}
$$

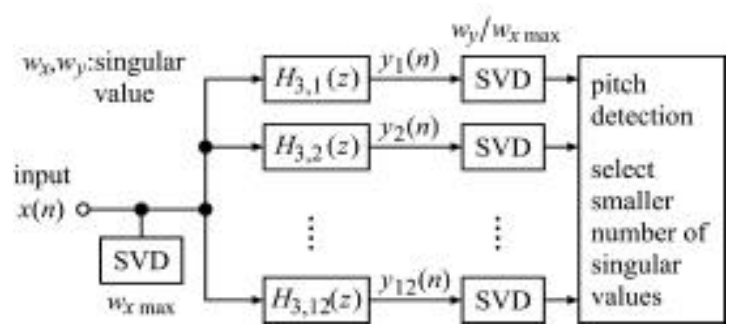

Fig.3 Pitch detection system using parallel connected comb filters and SVD.

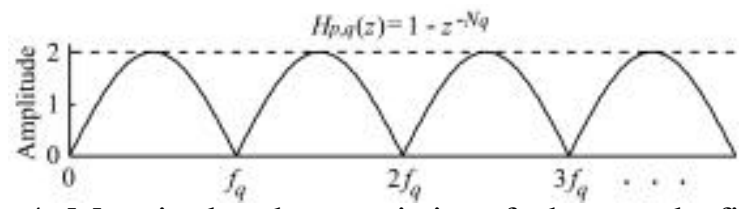

Fig.4 Magnitude characteristic of the comb filter $H_{p, q}(z)=1-z^{-N_{q}}$.
This comb filter has the frequency characteristic as shown in Fig. 4 and can eliminate the pitch and its harmonic frequency components, i.e., q pitch name. The pitch detection system consists of these twelve comb filters connected in parallel as shown in Fig.5(a) in the case of the input range between octaves 3 to 5 . For example, when a polyphonic sound of $\left(C_{3}+E_{3}+G_{3}\right)$ is entered into this system, the outputs $y_{1}(n)\left(C_{3}\right), y_{5}(n)\left(E_{3}\right)$ and $y_{8}(n)\left(G_{3}\right)$ has smaller frequency components than other outputs. Then it is expected that the output amplitude of these filters are smaller than other ones. When an input sound is single tone, the output of the corresponding comb filter shows zero amplitude. But, when an input is a polyphony, the comb filter outputs do not become zero. And so we detect the comb filter showing the minimum output as a candidate of the comb filters corresponding to the input pitches. The minimum output is determined by detecting the minimum output $y_{k \max }(n)$ in the twelve comb filters, where $y_{k \max }(n)$ shows the maximum output value in the some constant period of the comb filter output $y_{k}(n)$ for k pitch name. As the next step, we connect the comb filter showing the minimum output and the other parallel connected comb filters in cascade as shown in Fig.5(b). This process is continued until we can obtain a zero output.

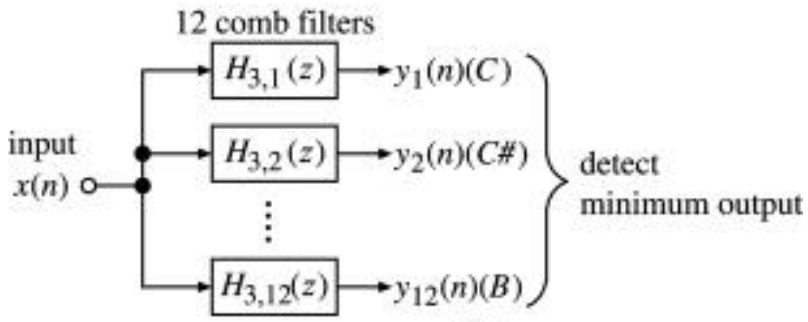

(a)

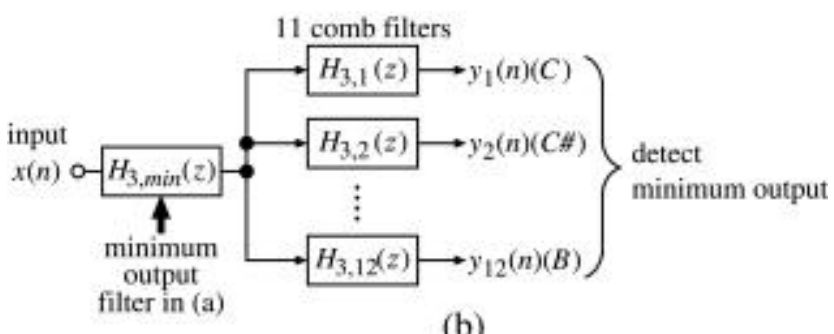

(b)

Fig.5 Pitch detection system using parallel connected comb filters and minimum output. 


\section{Simulation Results using Artificial Musical Sounds}

A musical sound has complex frequency components, and their amplitudes and phases corresponding to the musical instruments and their tones. To consider the feature of the proposed method, we use the following artificial musical sound that has $r$ tones and six harmonic components in each tone,

$x(n)=\sum_{j=1}^{r} \sum_{k=1}^{6} A_{j k} \cos \left(\left(2 \pi k f_{j} n / f_{s}\right)+\phi_{j k}\right)$

where $f_{s}$ is a sampling frequency and $f_{j}$ is a pitch frequency for $\mathrm{j}$ tone. For double and triple tones, we examined $66\left({ }_{12} C_{2}\right)$ and $220\left({ }_{12} C_{3}\right)$ chords of the combination of twelve pitch names $(\mathrm{C}, \mathrm{C} \#, \ldots, \mathrm{B})$ in octave 3 only, and $630\left({ }_{36} C_{2}\right)$ and 7,140 $\left({ }_{36} C_{3}\right)$ between octaves 3 to 5 . For each chord, we made $10^{4}$ inputs changing $A_{j k}$ and $\phi_{j k}$ at random in the range of $0 \leq A_{j k} \leq 1.0, \quad 0 \leq \phi_{j k} \leq 2 \pi$. But when the chords are triple tones and between octaves 3 to 5 , we made $10^{2}$ inputs.

We detect the maximum amplitude $y_{k \max }(n)$ in the period of $100 \mathrm{~ms}$ output for each comb filter, and then we select the comb filters showing the smaller $y_{k \max }(n)$ to detect the input pitches. For double tones, first we select the comb filter with the minimum output $y_{k \max }(n)$ in twelve comb filters connected in parallel as shown in Fig.5(a). Second, we connect the first selected comb filter and the other parallel eleven comb filters in cascade as shown in Fig.5(b). If we can obtain the comb filter showing zero output, we can detect two pitches correctly. For triple tones, we continue this process again. We select the comb filter showing the minimum output in eleven comb filters, and then set the second selected comb filter before the first selected comb filter and connect these filters to the other parallel ten comb filters in cascade. If we can select the two correct comb filters, we can get the comb filter showing zero output in ten comb filters connected in parallel.

By these processing, we can know twelve pitch names only and then we must decide the octave of these detected pitch names. We can decide the octave of these pitch names by changing the order of the comb filters [8][9]. Tables 1 and 2 show these results. We also show the results when we use only the detection system shown in Fig.5(a). For example, in the case of double tones, we select the comb filter
Table 1 Pitch detection probability (double tones)

\begin{tabular}{cccc}
\hline \hline system & input & octave 3 & in octaves 3 to 5 \\
\hline & one pitch & $99.6 \%$ & $83.6 \%$
\end{tabular}

Fig.5(a) two pitches $88.8 \% \quad 48.5 \%$

Fig.5(b) two pitches $98.3 \% \quad 69.3 \%(79.9 \%)$

Table 2 Pitch detection probability (triple tones)

\begin{tabular}{llcc}
\hline \hline system & input & octave 3 & in octaves 3 to 5 \\
\hline \multirow{3}{*}{ Fig.5(a) } & one pitch & $93.3 \%$ & $76.8 \%$ \\
& two pitches & $72.3 \%$ & $46.0 \%$ \\
& three pitches & $33.3 \%$ & $17.1 \%$ \\
\hline Fig.5(b) & three pitches & $91.1 \%$ & $33.9 \%(54.2 \%)$ \\
\hline
\end{tabular}

showing the minimum output or two comb filters with the smaller outputs. We show the probabilities when the comb filter with the minimum output is corresponding to one of two pitches and the selected two comb filters both are correct. When the chords are in the octave 3 only, we could detect the pitches with the probabilities of $98.3 \%$ and $91.1 \%$ for double and triple tones, respectively. For the chords between octaves 3 to 5, we could obtain the probabilities of $69.3 \%(79.9 \%)$ and $47.8 \%(54.2 \%)$ for double and triple tones, respectively.

In the case where the input pitches are between octaves 3 to 5 , we have the chords of the same pitch names of different octaves, e.g., $C_{3}$ and $C_{4}$, and the consonance, e.g., $C_{3}$ and $G_{4}$. The numbers in brackets in Tables 1 and 2 show the probabilities in the case excluding these chords. Naturally, we must distinguish these chords as the next step by the different method that now we are considering.

\section{Experimental Results using Actual Musical Sounds}

Fig.6 (a) shows a test score used in the experiment. This score was played by two different musical instruments, i.e., clarinet and violin. Fig.6 (c) and (d) show the results of pitch detection using the proposed method and the SVD method, respectively. These results are almost correct, but the proposed method has smaller computational complexity than the SVD method by the order of $10^{3}$. 


\section{Conclusions}

We proposed a simple pitch detection method noticing the minimum output in the twelve comb filters connected in parallel. Detecting the smaller outputs, we could reduce the computational complexity that was the problem in the parallel comb filter method using the SVD processing. We could obtain the pitch detection probability of $98.3 \%$ for double tones and

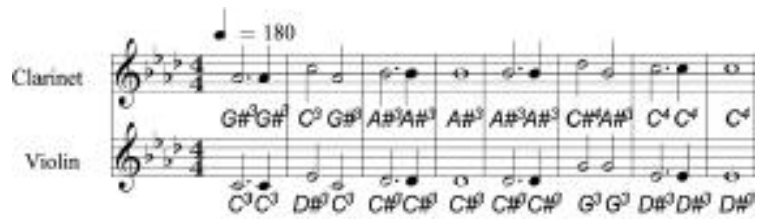

(a) test score: It's a small world

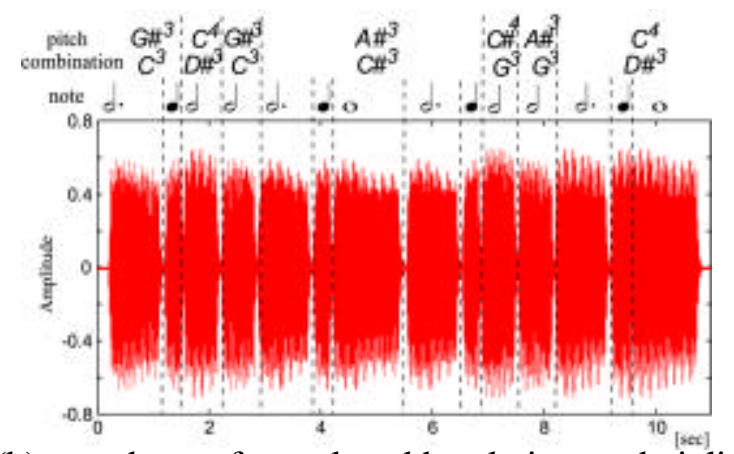

(b) sound waveform played by clarinet and violin

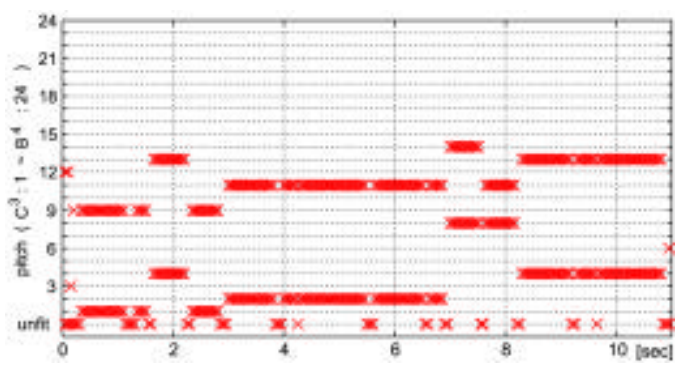

(c) pitch detection results of the proposed method

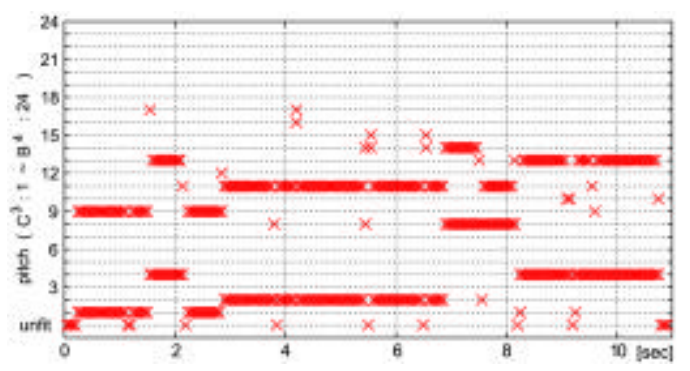

(d) pitch detection results of the SVD method

Fig.6 Pitch detection results. (c) the proposed method and (d) the SVD method.
91.1\% for triple tones in octave 3 only. As a future research, we must consider the method to distinguish the tones that are the same pitch name in the different octaves or consonance.

\section{References:}

[1] M. Piszczalski and B. A. Galler, "Automatic music transcription," Computer Music Journal, vol. 1, no. 4, pp. 24-31,1977.

[2]C. Roads, "Research in music and artificial intelligence," ACM computing Surveys, vol. 17, no. 2 pp. 163-190,1985.

[3]H. Katayose and S. Inoguchi, "Intelligent music transcription system," Trans. of Artificial Intelligence of Japan, vol. 5, no. 1 pp.59-66, 1990.

[4]J. A. Moorer, "Signal processing aspects of computer music : a survey," Proceedings of IEEE, pp. 1108-1137, 1977.

[5]W. J. Pielemeiier and G. H. Wakefield, "A high-resolution time--frequency representation of music al instrument signals," J. Acoust. Soc. Am. vol. 99, no. 4, Pt. 1, pp. 2382-2396, 1996.

[6]S. Foster, J. Rockmore, W. Schloss, "Toward an intelligent editor of digital audio: signal processing methods,"' Computer Music Journal, vol. 6, no. 1, pp. 142-61, 1982.

[7]T.Umemoto and N.Aoshima, "The adaptive spectrum analysis for transcription," Trans. of Society of Instrument and Control Engineering of Japan, vol.28, no.5, pp.619-626, 1992.

[8]T.Miwa,Y.Tadokoro and T.Saito, "The pitch estimation of different musical instruments sounds using comb filters for transcription," Trans. of IEICE of Japan, vol.J81-D-II, no.9, pp.1965-1974, 1998.

[9]Y.Tadokoro and T.Miwa, "Musical pitch and instrument estimation of polyphony using comb filters for transcription," CSCC200, Advances in Physics, Electronics and Signal Processing Applications, pp.315-319, 2000.

[10]Y.Tadokoro, W.Matsumoto and M.Yamaguchi, "Pitch detection of musical sounds using adaptive comb filters controlled by time delay, " IEEE International Conference on Multimedia and Expo, (to be accepted), Lausanne, Switzerland, 2002.

[11]M.Yamaguchi and Y.Tadokoro, "Pitch detection of song sounds using parallel connected comb filters and singular value decomposition, " IEEE International Symposium on Intelligent signal processing and communication systems (ISPACS), (to be submitted), Taiwan, 2002. 